\title{
REVISIÓN SOBRE EL ESTADO ACTUAL DE LA CALIDAD DE VIDA EN PERSONAS SOBREVIVIENTES A ICTUS do
}

\author{
Gloria Johanna Montaña Mogollón ㅁ, \& Elsa Fernanda Siabato \\ $\operatorname{Macias}^{1}{ }^{1}{ }^{2}$
}

Universidad de Boyacá, Tunja, Colombia.

\section{RESUMEN}

El presente artículo tuvo como objetivo conocer los hallazgos sobre la calidad de vida (CV) en pacientes sobrevivientes a ictus, mediante la revisión de estudios empíricos publicados en el periodo de 2012 a 2017, en las bases de datos de Sciencedirect y Proquest. Se llevó a cabo un estudio descriptivo de tipo documental, en el que se utilizaron como descriptores de búsqueda las palabras: calidad de vida, ictus y ACV. Se identificaron inicialmente 1679 artículos, de los cuales se seleccionaron 44 que cumplieron con los criterios de inclusión. De cada uno de los artículos se analizó el diseño metodológico, las características de los participantes, los instrumentos de medición de la $\mathrm{CV}$, los principales resultados obtenidos y las limitaciones reportadas. De los 44 artículos revisados el $97 \%$ eran de corte cuantitativo, siendo el diseño observacional transversal con alcance relacional el más prevalente. El principal tema abordado en las investigaciones fue el de los factores asociados a la CV; siendo la funcionalidad, el estado neurológico y la depresión, las variables predictivas más reportadas. Se espera que a futuro la investigación sobre el tema se realice a través de investigaciones mixtas o con diseños observacionales longitudinales, que incluyan participantes con edades inferiores a los 18 años, que realicen evaluaciones con escalas específicas, y que profundicen sobre el efecto de variables cognitivas, psicológicas y del proceso de cuidado en la Calidad de vida.

Palabras Claves

calidad de vida, accidente cerebrovascular, revisión

\begin{abstract}
The objective of this article was to know the findings about the quality of life (QL) in surviving patients through the review of empirical studies published in the period from 2012 to 2017 in the databases of Sciencedirect and Proquest. A documentary descriptive study was conducted, in which the following words were used as search descriptors: quality of life, ictus, and ACV were used for the research. 1679 articles were initially identified, 44 of those were selected based on their fulfillment of the inclusion criteria. The articles were analyzed according to the methodological design, the characteristics of the participants, the measurement instruments, the main results obtained and the limitations reported. A total of 44 studies were found, o $97 \%$ were quantitative, with the observational cross-sectional design with the most prevalent relational scope. The main issue addressed in the studies was the factors associated with CV; being functionality, neurological status and depression, the most reported predictive variables. It is expected that in the future, the research about the topic will be made through mixed investigation or with observational longitudinal designs, that include participants younger than 18; should perform assessments with specific scales, and should delve into the effect of cognitive and psychological variables, and care process in the life quality.
\end{abstract}

\section{Keywords}

quality of life, stroke, review

\footnotetext{
1 Correspondence about this article should be addressed to Elsa Fernanda Siabato Macias: efsiabato@uniboyaca.edu.co

2 Conflicts of Interest: The authors declare that the research was conducted in the absence of any commercial or financial relationships that could be construed as a potential conflict of interest.
} 


\section{REVIEW OF THE CURRENT STATUS OF THE QUALITY OF LIFE IN STROKE SURVIVORS}

\section{Introducción}

El Ictus, también conocido como Enfermedad Cerebrovascular (ECV) o Accidente Cerebrovascular (ACV), es una condición neurológica asociada a daños en los vasos sanguíneos del cerebro, y al rápido desarrollo de signos neurológicos focales. De acuerdo con datos epidemiológicos el ictus es considerado un problema de salud pública, teniendo en cuenta que para el año 2016, se registraron 5.5 millones de muertes y 116. 4 millones de años de vida ajustados por discapacidad, siendo la segunda causa de muerte a nivel mundial (GBD 2016 Stroke Collaborators, 2019). Cabe aclarar que la epidemiología del ictus ha tenido cambios, tradicionalmente se asocia a personas de edad avanzada, sin embargo, en la actualidad ha aumentado la incidencia en menores de 45 años por la presencia de factores de riesgo clásicos como el consumo de tabaco, hipertensión arterial, obesidad y diabetes (González-Gómez, et al., 2016).

El momento vital en el que se presenta el evento puede suscitar cambios en los planes y retos hacia el futuro en la persona que padece la enfermedad y también en su grupo familiar, por lo cual se deben hacer reajustes, ante lo que para muchos es una crisis. Las consecuencias para la salud y las diversas limitaciones en diferentes áreas de desempeño (Jaracza et al., 2014), han motivado a los investigadores a realizar estudios orientados al análisis de la Calidad de Vida (CV) en sobrevivientes a Ictus.

El constructo de $\mathrm{CV}$ es amplio, dinámico, complejo, multidimensional y multifactorial, que tiene una alta relevancia en escenarios sociales y de salud. Urzúa \& Caqueo (2012) señalan que la CV es el "nivel percibido de bienestar derivado de la evaluación que realiza cada persona de elementos objetivos y subjetivos en distintas dimensiones de su vida" (p.65), en coherencia con lo anterior, la CV comprende la evaluación de las facetas social, psicológica, física, nivel de independencia y espiritualidad (The World Health Organization Quality of Life Assessment group, WHOQOL,1998).

De otra parte, cuando se hace referencia exclusiva a temas de salud, en este caso al diagnóstico de ictus, el concepto de CV tiene otras connotaciones debido a la influencia que tienen la presencia de procesos patológicos y el efecto del tratamiento (Robles et al., 2016; Urzúa, 2010; Urzúa \& Caqueo, 2012). Específicamente para el ictus, en el cual las secuelas suelen ser duraderas, difíciles de cuantificar y de tratar; la evaluación de la CV 
se ha convertido en un constructo relevante para el análisis de las consecuencias de la enfermedad y de los efectos de los diversos tratamientos en el bienestar de las personas (Hohmann et al., 2010; Rachpukdee et al., 2013).

Por consiguiente, es de interés para el presente estudio describir el estado actual de las investigaciones sobre $\mathrm{CV}$ en sobrevivientes a ictus durante los últimos 5 años, con el propósito de develar los avances y los vacíos empíricos que se encuentran en el estudio de dicho fenómeno. Cabe resaltar que se halló un estudio de revisión sobre los factores asociados a la CV post ictus (Mesa et al.,2017), sin embargo, surge la necesidad de realizar un análisis más detallado, no solo de los factores asociados, sino de otros aspectos como los diseños y alcances de las investigaciones, los objetivos desarrollados, las características de los participantes, los instrumentos de medición, las conclusiones principales y las limitaciones de los estudios. En síntesis, se espera generar un panorama actual y oportuno que permita orientar los lineamientos de próximas investigaciones que deseen abordar el tema, dada la importancia de la valoración de la CV en la definición e implementación de intervenciones adecuadas para este grupo poblacional (Jaracza et al., 2014).

\section{Método}

Se realizó un estudio descriptivo de tipo documental, el cual consiste en un análisis de fuentes primarias sobre un determinado tema, mediante la aplicación de métodos y técnicas de búsqueda y de procesamiento de la información, con el fin identificar relaciones, diferencias, posturas o estado actual del conocimiento respecto al fenómeno objeto de estudio, bajo condiciones de sistematización, coherencia y argumentación (Bernal, 2010). Para el presente estudio se establecieron como unidades de análisis, los artículos originales publicados en las bases de datos ScienceDirect y Proquest, durante el periodo comprendido entre 2012 y 2017. Se seleccionó la base de datos ScienceDirect, ya que, es la base líder de Elsevier, que combina publicaciones científicas y de salud en texto completo, con más de 2,500 títulos de revistas (Elsevier, 2020), por su parte, Proquest, cuenta con cinco bases de datos sobre temas de la disciplina psicológica y de la salud (Proquest, 2020). Se usaron como descriptores de búsqueda las palabras calidad de vida, ictus y ACV, tanto en español como en inglés.

Se consideraron los siguientes criterios de inclusión: artículos originales publicados de enero de 2012 a diciembre de 2017, en inglés o español, de corte cuantitativo o cualitativo y cuyos resultados abordaron la calidad de vida de los 
sobrevivientes a ictus. Se plantearon como criterios de exclusión: artículos duplicados o estudios que contuvieran los descriptores pero cuyo objetivo estuviese centrado en otra temática.

\section{Procedimento}

Se realizó la búsqueda en las bases de datos ScienceDirect y Proquest, se identificaron a partir de los descriptores todos los artículos empíricos publicados entre el 2012 y el 2017, seleccionándose 44 artículos que cumplieron con los criterios de inclusión, 35 fueron hallados en la base de datos ScienceDirect, siete en Proquest y dos registrados en ambas bases de datos (ver figura 1), posteriormente, se construyó un protocolo de registro de la información hallada, clasificándose los estudios de acuerdo al diseño metodológico, participantes, principales conclusiones y limitaciones; además se reportó por cada artículo los siguientes datos: autor, año, país, objetivo, muestra e instrumento de medición de la calidad de vida.
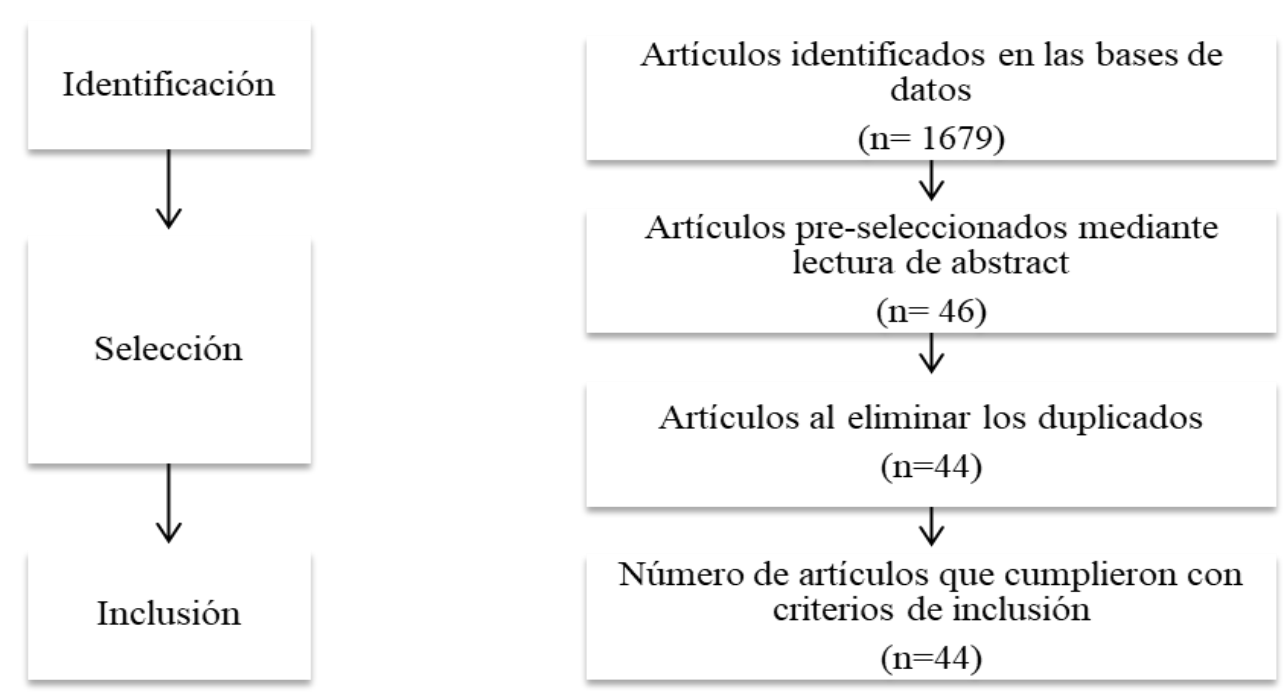

Figura 1. Flujograma de selección de los artículos

\section{Resultados}

A continuación, se muestran los hallazgos obtenidos. En primer lugar, se encuentra el análisis de los diseños de investigación, seguido de las características de la población, los instrumentos de medición, las conclusiones principales y finalmente, las limitaciones de los estudios. 


\section{Diseños de investigación}

Con el fin de identificar los enfoques de investigación y los diseños metodológicos más prevalentes en los estudios, en la tabla 1 se muestra el análisis de frecuencia.

Tabla 1.

Distribución según el diseño metodológico

\begin{tabular}{cccccccc}
\hline $\begin{array}{c}\text { Tipo de } \\
\text { investigación }\end{array}$ & $\mathrm{N}$ & $\begin{array}{c}\text { Diseño según el } \\
\text { propósito }\end{array}$ & $\mathrm{n}$ & $\begin{array}{c}\text { Diseño según } \mathrm{n}^{\circ} \text { de } \\
\text { mediciones }\end{array}$ & $\mathrm{n}$ & Alcance & $\mathrm{n}$ \\
\hline Cuantitativo & 42 & Observacional & 40 & Transversal & 23 & Descriptivo & 1 \\
& & & & & & $\begin{array}{c}\text { Relacional } \\
\text { Psicométrico }\end{array}$ & 20 \\
\cline { 3 - 7 } & & & & Longitudinal & 17 & Descriptivo & 1 \\
& & & & & Relacional & 12 \\
& & & & & Explicativo & 3 \\
& & & & & Psicométrico & 1 \\
\cline { 3 - 7 } & & Experimental & 2 & Longitudinal & 2 & Explicativo & 2 \\
\hline Mixto & 1 & Secuencial & 1 & Transversal & 1 & Relacional & 1 \\
\hline Cualitativo & 1 & Fenomenológico & 1 & -- & -- & Descriptivo & 1 \\
\hline
\end{tabular}

Por otra parte, se identificó que la mayoría de los estudios (75\%) presentaron un alcance relacional, en los cuales se determinaron las variables asociadas a la calidad de vida en sobrevivientes a ictus, así como su efecto en la variable dependiente (CV) a partir de análisis multivariados. Debido a que la mayoría de investigaciones fueron de corte transversal y no se logra establecer una secuencia temporal de las variables evaluadas (causa-efecto) (Bradford-Hill, 1992), se dificulta determinar relaciones causales, por consiguiente, es menor el porcentaje de artículos que obtienen un alcance explicativo (11\%). Respecto a investigaciones de corte psicométrico, se encontró que el 7\% de las pesquisas realizaron adaptaciones o validaciones de instrumentos frecuentemente usados en la evaluación de la CV de sobrevivientes a ictus.

\section{Características de las poblaciones}

Para continuar con el análisis, en la tabla 2, se describen los artículos seleccionados en orden descendente de acuerdo a su fecha de publicación, y se puntualiza en los objetivos y las características de la muestra.

Tabla 2

Descripción de los artículos relacionados con CV en sobrevivientes a un ACV

\begin{tabular}{rrrccl}
\hline $\mathrm{N}^{\circ}$ & Autor & Año & País & Muestra & \multicolumn{1}{c}{ Objetivo } \\
\hline $\begin{array}{l}\text { Dąbrowska- } \\
\text { Bender et al. }\end{array}$ & 2017 & Polonia & $\begin{array}{l}\text { un pacientes con } \\
\text { unimer ictus } \\
\text { isquémico. }\end{array}$ & $\begin{array}{l}\text { Evaluar la Calidad de vida de } \\
\text { Pacientes quienes sufrieron de } \\
\text { ictus isquémico con respecto a }\end{array}$ \\
\hline
\end{tabular}




\begin{tabular}{|c|c|c|c|c|c|}
\hline & & & & & $\begin{array}{l}\text { varias áreas de la vida incluyendo } \\
\text { factores clínicos } \\
\text { psicoemocionales }\end{array}$ \\
\hline 2 & $\begin{array}{l}\text { Ezeugwu \& } \\
\text { Manns }\end{array}$ & 2017 & Canadá & $\begin{array}{l}30 \text { personas. con } \\
\text { ictus } \\
\text { hemorrágico o } \\
\text { isquémico con 2- } \\
4 \text { semanas } \\
\text { posteriores al alta } \\
\text { de un centro de } \\
\text { rehabilitación de } \\
\text { accidente } \\
\text { cerebrovascular. }\end{array}$ & $\begin{array}{l}\text { Describir la duración del sueño, la } \\
\text { conducta sedentaria, la actividad } \\
\text { física y la } \mathrm{CV} \text {, así como su } \\
\text { asociación con factores } \\
\text { demográficos y clínicos dentro del } \\
\text { primer mes después de la } \\
\text { rehabilitación del ictus. }\end{array}$ \\
\hline 3 & Kim et al. & 2017 & $\begin{array}{c}\text { República de } \\
\text { Korea }\end{array}$ & $\begin{array}{l}214 \text { pacientes } \\
\text { con accidente } \\
\text { cerebrovascular } \\
\text { por primera vez } \\
\text { ingresados en } \\
\text { una unidad de } \\
\text { rehabilitación. }{ }^{\text {a }}\end{array}$ & $\begin{array}{l}\text { Investigar la relación entre el } \\
\text { insomnio y la CVRS durante la } \\
\text { etapa temprana de la } \\
\text { rehabilitación del accidente } \\
\text { cerebrovascular. }\end{array}$ \\
\hline 4 & Boulus et al. & 2017 & Canadá & $\begin{array}{l}94 \\
\text { sobrevivientes a } \\
\text { ictus, de los } \\
\text { cuales } 23 \text { fueron } \\
\text { diagnosticados } \\
\text { con Síndrome de } \\
\text { piernas } \\
\text { inquietas. }{ }^{c}\end{array}$ & $\begin{array}{l}\text { Evaluar el efecto del síndrome de } \\
\text { piernas inquietas en la CV de } \\
\text { personas sobrevivientes a un } \\
\text { ACV. }\end{array}$ \\
\hline 5 & Peixoto et al. & 2017 & Portugal & $\begin{array}{l}51 \text { pacientes de } \\
\text { dos clínicas de } \\
\text { rehabilitación } \\
\text { física para } \\
\text { pacientes } \\
\text { ambulatorios, } \\
\text { con antecedentes } \\
\text { de un primer } \\
\text { episodio de } \\
\text { ACV. b, c }\end{array}$ & $\begin{array}{l}\text { Determinar las variables } \\
\text { predictivas de CV y sus dominios } \\
\text { en pacientes portugueses durante } \\
\text { el proceso de rehabilitación física } \\
\text { después } \\
\text { primer ictus }\end{array}$ \\
\hline 6 & Persson et al. & 2017 & Suecia & $\begin{array}{l}248 \\
\text { sobrevivientes y } \\
\text { su pareja; así } \\
\text { como r } 245 \\
\text { controles y su } \\
\text { pareja. }^{c}\end{array}$ & $\begin{array}{l}\text { Describir la CVRS y la CV } \\
\text { ajustada a años de vida tanto en } \\
\text { sobrevivientes a ictus como en las } \\
\text { parejas de los sobrevivientes. }\end{array}$ \\
\hline 7 & Sarfo et al. & 2017 & Ghana & $\begin{array}{l}200 \\
\text { sobrevivientes a } \\
\text { ictus. }^{c}\end{array}$ & $\begin{array}{l}\text { Evaluar la prevalencia y los } \\
\text { predictores de la depresión en una } \\
\text { muestra por conveniencia de } \\
\text { sobrevivientes de ACV de Ghana }\end{array}$ \\
\hline 8 & Theeke et al. & 2017 & China & $\begin{array}{l}6 \text { sobrevivientes } \\
\text { adultos de ACV } \\
(5 \text { isquémicos } 1 \\
{\text { hemorrágico })^{b}}^{b}\end{array}$ & $\begin{array}{l}\text { Describir la estructura del } \\
\text { significado en la experiencia de } \\
\text { sobrevivir a un ictus para los } \\
\text { adultos que viven en los } \\
\text { Apalaches. }\end{array}$ \\
\hline 9 & Andrew et al. & 2016 & Australia & $\begin{array}{l}173 \\
\text { sobrevivientes de } \\
\mathrm{ACV}^{\mathrm{b}}\end{array}$ & $\begin{array}{l}\text { Comprender si la calidad de vida } \\
\text { relacionada con la } \\
\text { salud (CVRS) entre } 90 \text { y } 180 \text { días } \\
\text { después del accidente } \\
\text { cerebrovascular se asocia con las } \\
\text { necesidades insatisfechas a largo } \\
\text { plazo. }\end{array}$ \\
\hline
\end{tabular}




\begin{tabular}{|c|c|c|c|c|c|}
\hline 10 & $\begin{array}{l}\text { Chuluunbaatar } \\
\text { et al. }\end{array}$ & 2016 & Mongolia & $\begin{array}{l}155 \text { pacientes } \\
\text { con ACV por } \\
\text { primera vez y sus } \\
88 \quad \text { cuidadores } \\
\text { informales. }{ }^{\text {a }}\end{array}$ & $\begin{array}{l}\text { Describir los cambios en la CdV y } \\
\text { determinar los factores asociados } \\
\text { con la CdV tanto para los } \\
\text { pacientes con accidente } \\
\text { cerebrovascular como para sus } \\
\text { cuidadores informales durante el } \\
\text { primer año después del accidente } \\
\text { cerebrovascular. }\end{array}$ \\
\hline 11 & Crichton et al. & 2016 & Londres & $\begin{array}{l}262 \\
\text { sobrevivientes a } \\
\text { ictus por } 15 \text { años } \\
\text { c }\end{array}$ & $\begin{array}{l}\text { Estimar los resultados a largo } \\
\text { plazo del ACV en múltiples } \\
\text { dominios de la salud } \\
\text { (supervivencia, funcional, } \\
\text { cognitivo, calidad de vida y salud } \\
\text { mental) hasta } 15 \text { años después del } \\
\text { accidente cerebrovascular. }\end{array}$ \\
\hline 12 & Karube et al. & 2016 & Japón & $\begin{array}{l}53 \text { pacientes } \\
\text { ambulatorios } \\
\text { sobrevivientes de } \\
\text { ACV (46 de tipo } \\
\text { isquémico y } 7 \\
\text { hemorrágico). }{ }^{c}\end{array}$ & $\begin{array}{l}\text { Investigar las relaciones entre tres } \\
\text { dominios de CV (salud física, } \\
\text { salud psicológica, y relaciones } \\
\text { sociales y ambientales) durante el } \\
\text { período de posthospitalización en } \\
\text { pacientes con accidente } \\
\text { cerebrovascular }\end{array}$ \\
\hline 13 & Khalid et al. & 2016 & Pakistán & $\begin{array}{lr}350 \quad \text { diadas } \\
\text { pacientes }^{\text {cuidadores. }}{ }^{c} & y\end{array}$ & $\begin{array}{l}\text { Describir la } \mathrm{CV} \text { de los } \\
\text { sobrevivientes de } \\
\text { cuidadores }\end{array}$ \\
\hline 14 & Pinedo et al. & 2016 & España & $\begin{array}{l}157 \text { pacientes } \\
\text { que ingresaron } \\
\text { en la fase } \\
\text { subaguda tras un } \\
\text { ictus en } 2 \\
\text { Servicios de } \\
\text { Rehabilitación. }{ }^{\text {b }}\end{array}$ & $\begin{array}{l}\text { Describir la CV de los pacientes a } \\
\text { los } 6 \text { meses de evolución del ictus } \\
\text { y su relación con variables } \\
\text { sociodemográficas, clínicas y } \\
\text { funcionales }\end{array}$ \\
\hline 15 & Chou & 2015 & Taiwan & $\begin{array}{l}134 \text { pacientes } \\
\text { con al menos } 6 \\
\text { meses de } \\
\text { evolución } \\
\text { atendidos en tres } \\
\text { centros de } \\
\text { rehabilitación. }{ }^{c}\end{array}$ & $\begin{array}{l}\text { Identificar los factores que se } \\
\text { relacionan en gran medida con la } \\
\text { CV post ictus }\end{array}$ \\
\hline 16 & Guajardo et al. & 2015 & Brasil & $\begin{array}{l}67 \text { pacientes un } \\
\text { mes y tres meses } \\
\text { después del } \\
\text { primer ictus } \\
\text { isquémico. }\end{array}$ & $\begin{array}{l}\text { Evaluar el impacto de los } \\
\text { síntomas depresivos en } 1 \text { y } 3 \\
\text { meses después del ACV en la } \\
\text { CVRS }\end{array}$ \\
\hline 17 & Gillard et al. & 2015 & $\begin{array}{l}\text { EEUU- } \\
\text { California }\end{array}$ & $\begin{array}{l}40 \\
\text { sobrevivientes a } \\
\text { ictus isquémico }{ }^{\text {b }}\end{array}$ & $\begin{array}{l}\text { Examinar el impacto de la } \\
\text { espasticidad en la CV de los } \\
\text { sobrevivientes a ictus. A los } 3 \\
\text { meses post ictus, } 1 \text { y } 2 \text { años } \\
\text { después. }\end{array}$ \\
\hline 18 & Leno et al. & 2015 & España & $\begin{array}{l}75 \\
\text { sobrevivientes a } \\
\text { un ACV. }^{c}\end{array}$ & $\begin{array}{l}\text { Valorar la CV a largo plazo en } \\
\text { pacientes que sufrieron un ictus y } \\
\text { determinar aquellos factores } \\
\text { relacionados con la misma. }\end{array}$ \\
\hline 19 & De Bruijin et al. & 2015 & Países Bajos & $\begin{array}{l}170 \text { pacientes } \\
\text { jóvenes } \\
\text { (menores de } 50\end{array}$ & $\begin{array}{l}\text { Determinar los factores médicos y } \\
\text { psicológicos que influyen en la } \\
\mathrm{CV} \text { en jóvenes pacientes con }\end{array}$ \\
\hline
\end{tabular}




\begin{tabular}{|c|c|c|c|c|c|}
\hline & & & & $\begin{array}{lr}\text { años) con ictus } \\
\text { isquémico, } \\
\text { ingresados en un } \\
\text { Hospital } & \text { de } \\
\text { Países } & \text { Bajos, } \\
\text { entre } 2000 & \text { y } \\
2010 ; & \text { y } \\
\text { controles. }^{c} & \\
\end{array}$ & $\begin{array}{l}\text { accidente cerebrovascular, } \\
\text { después del seguimiento a largo } \\
\text { plazo (en promedio } 4.9 \text { años) }\end{array}$ \\
\hline 20 & Tran et al. & 2015 & Vietman & $\begin{array}{l}108 \text { pacientes } \\
\text { con un primer } \\
\text { accidente } \\
\text { cerebrovascular }^{\mathrm{b}}\end{array}$ & $\begin{array}{l}\text { Evaluar la confiabilidad y validez } \\
\text { de los test EQ-5D y el DHP para } \\
\text { cuidadores y para pacientes }\end{array}$ \\
\hline 21 & $\begin{array}{l}\text { Van Mierlo et } \\
\text { al. }\end{array}$ & 2015 & Países bajos & $\begin{array}{l}287 \text { pacientes } \\
\text { con diagnóstico } \\
\text { de ACV } \\
\text { (hemorrágico, } \\
\text { isquémico o } \\
\text { intracerebral). b }\end{array}$ & $\begin{array}{l}\text { Examinar las asociaciones entre } \\
\text { cogniciones de la enfermedad y } \\
\text { satisfacción con la vida a los dos } \\
\text { meses y dos años después del } \\
\text { ACV }\end{array}$ \\
\hline 22 & Cortez et al. & 2014 & EEUU & $\begin{array}{l}43 \\
\text { sobrevivientes de } \\
\mathrm{ACV} \text { isquémico } \\
\text { agudo }{ }^{\text {a }}\end{array}$ & $\begin{array}{l}\text { Determinar la calidad de vida en } \\
\text { pacientes que recibieron Terapia } \\
\text { para el accidente cerebrovascular } \\
\text { isquémico agudo }\end{array}$ \\
\hline 23 & Cumming, et al. & 2014 & Australia & $\begin{array}{l}56 \text { pacientes que } \\
\text { ingresaron en la } \\
\text { unidad de ictus } \\
\text { agudo }^{\text {a }}\end{array}$ & $\begin{array}{l}\text { Determinar la importancia de los } \\
\text { déficits en diferentes dominios } \\
\text { cognitivos sobre la CV }\end{array}$ \\
\hline 24 & De Lima et al. & 2014 & Brasil & $\begin{array}{l}\text { Grupo } r \\
\text { personas con } \\
\text { ACV y que } \\
\text { tienen cuidador } \\
\text { (44), grupo sin } \\
\text { cuidadores (39), } \\
\text { grupo de los } \\
\text { cuidadores }(44) \text { y } \\
\text { un grupo de } \\
\text { referencia }(83)^{\text {c }}\end{array}$ & $\begin{array}{l}\text { Comparar la CV de los pacientes } \\
\text { y los cuidadores. }\end{array}$ \\
\hline 25 & Dhamoon et al. & 2014 & EEUU & $\begin{array}{l}2870 \text { pacientes } \\
\text { con síndrome de } \\
\text { apoplejía lacunar } \\
\text { clínica o ataque } \\
\text { isquémico } \\
\text { transitorio } \\
\text { subcortical (AIT) } \\
\text { b }\end{array}$ & $\begin{array}{l}\text { Describir el curso y los } \\
\text { predictores de la calidad de vida } \\
\text { después del accidente } \\
\text { cerebrovascular lacunar. }\end{array}$ \\
\hline 26 & $\begin{array}{l}\text { McDonnell et } \\
\text { al. }\end{array}$ & 2014 & Australia & $\begin{array}{l}13 \text { adultos } \\
\text { mayores sanos, } \\
17 \text { adultos con } \\
\text { ictus que asistían } \\
\text { a grupos de } \\
\text { ejercicio, y } 10 \\
\text { adultos con ictus } \\
\text { que no realizaban } \\
\text { estas actividades. } \\
\text { c }\end{array}$ & $\begin{array}{l}\text { Investigar si la actividad física } \\
\text { regular se asocia a un mejor } \\
\text { estado de ánimo y calidad de vida }\end{array}$ \\
\hline 27 & Tang et al. & 2014 & China & $\begin{array}{l}391 \text { pacientes de } \\
\text { una unidad de } \\
\text { ACV aguda. } \\
\text { Accidente. }{ }^{\text {a }}\end{array}$ & $\begin{array}{l}\text { Examinar las diferencias en la } \\
\text { calidad de vida relacionada con la } \\
\text { salud (CVRS) en los } \\
\text { sobrevivientes de accidente } \\
\text { cerebrovascular con y sin apatía. }\end{array}$ \\
\hline
\end{tabular}




\begin{tabular}{|c|c|c|c|c|c|}
\hline 28 & Wu et al. & 2014 & China & $\begin{array}{l}386 \text { pacientes } \\
\text { con } \mathrm{ACV}^{\mathrm{c}}\end{array}$ & $\begin{array}{l}\text { Evaluar el impacto del sexo en la } \\
\text { CVRS en sobrevivientes chinos } \\
\text { de ACV a los } 6 \text { meses después del } \\
\text { alta. }\end{array}$ \\
\hline 29 & Cruz-Cruz, et al. & 2013 & México & $\begin{array}{l}31 \text { pacientes con } \\
\text { un año de } \\
\text { evolución } \\
\text { después de un } \\
\text { ACV }^{\text {c }}\end{array}$ & $\begin{array}{l}\text { Evaluar la confiabilidad y validez } \\
\text { de la versión previa en español de } \\
\text { la escala SSQOL versión } 2.0\end{array}$ \\
\hline 30 & De Souza, et al. & 2013 & España & $\begin{array}{l}21 \text { pacientes con } \\
\text { enfermedad de } \\
\text { chagas y un } \\
\text { diagnóstico } \\
\text { previo de ACV. }\end{array}$ & $\begin{array}{l}\text { Investigar la correlación de la } \\
\text { discapacidad del accidente } \\
\text { cerebrovascular y los síntomas } \\
\text { depresivos con el rendimiento } \\
\text { funcional y la calidad de vida en } \\
\text { pacientes con ACV chagásico. }\end{array}$ \\
\hline 31 & Ellis et al. & 2013 & EEUU & $\begin{array}{l}666 \text { con historia } \\
\text { de apoplejía. }{ }^{c}\end{array}$ & $\begin{array}{l}\text { Evaluar la Calidad de vida post } \\
\text { ictus. }\end{array}$ \\
\hline 32 & Haghgoo et al. & 2013 & Irán & $\begin{array}{l}40 \\
\text { sobrevivientes a } \\
\text { ictus. }{ }^{c}\end{array}$ & $\begin{array}{l}\text { Examinar la relación entre las } \\
\text { actividades de la vida diaria y el } \\
\text { grado de depresión después del } \\
\mathrm{ACV} \text {, así como la CV en } \\
\text { sobrevivientes iraníes de ACV }\end{array}$ \\
\hline 33 & Huang et al. & 2013 & Taiwan & $\begin{array}{l}74 \\
\text { sobrevivientes a } \\
\text { primer ictus, sin } \\
\text { deficiencia } \\
\text { cognitiva, } \\
\text { ausencia de } \\
\text { espasticidad } \\
\text { excesiva en las } \\
\text { articulaciones de } \\
\text { la extremidad } \\
\text { superior afectada } \\
\text { a, b, c }\end{array}$ & $\begin{array}{l}\text { Identificar los factores predictivos } \\
\text { del cambio en la CV después de } \\
\text { una forma distribuida de terapia } \\
\text { inducida por restricción entre los } \\
\text { sobrevivientes de ACV }\end{array}$ \\
\hline 34 & Kim et al. & 2013 & $\begin{array}{l}\text { Korea del } \\
\quad \text { sur }\end{array}$ & $\begin{array}{l}151 \text { pacientes } \\
\text { con ictus } \\
\text { isquémico. }{ }^{b}\end{array}$ & $\begin{array}{l}\text { Investigar la influencia de los } \\
\text { rasgos de personalidad en la } \\
\text { calidad de vida tres meses después } \\
\text { de ictus. }\end{array}$ \\
\hline 35 & $\begin{array}{l}\text { Rachpukdee et } \\
\text { al. }\end{array}$ & 2013 & Tailandia & $\begin{array}{l}125 \\
\text { sobrevivientes de } \\
\text { ACV reclutados } \\
\text { de } 4 \text { hospitales } \\
\text { públicos. }{ }^{\text {c }}\end{array}$ & $\begin{array}{l}\text { Evaluar, comparar e identificar } \\
\text { predictores de calidad de vida } \\
\text { insatisfactoria en diferentes } \\
\text { dimensiones de los sobrevivientes } \\
\text { de ACV en } 1 \text { mes y } 3 \text { meses } \\
\text { después del ictus }\end{array}$ \\
\hline 36 & Soriano et al. & 2013 & España & $\begin{array}{l}95 \text { pacientes que } \\
\text { presentaron un } \\
\text { ictus en el último } \\
\text { año, que habían } \\
\text { permanecido } \\
\text { ingresados en } \\
\text { una Unidad de } \\
\text { Rehabilitación } \\
\text { Neurológica. }^{c}\end{array}$ & $\begin{array}{l}\text { Describir el perfil } \\
\text { sociodemográfico, clínico y la } \\
\text { CVRS, según la encuesta ECVI- } \\
38 \text { y su relación con las diferentes } \\
\text { características } \\
\text { sociodemográficas y clínicas }\end{array}$ \\
\hline 37 & Tang et al. & 2013 & China & 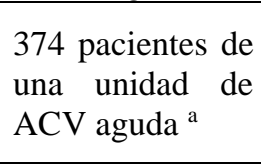 & $\begin{array}{l}\text { Examinar el impacto de la } \\
\text { ansiedad en la CVRS de los } \\
\text { sobrevivientes de accidentes } \\
\text { cerebrovasculares. }\end{array}$ \\
\hline 38 & Wong et al. & 2013 & China & $\begin{array}{l}100 \text { pacientes } \\
\text { con hemorragia } \\
\text { subaracnoidea }\end{array}$ & $\begin{array}{l}\text { Desarrollar una versión china de } \\
12 \text { ítems de Escala de calidad de }\end{array}$ \\
\hline
\end{tabular}




\begin{tabular}{|c|c|c|c|c|c|}
\hline & & & & $\begin{array}{l}\text { espontánea e } \\
\text { ingreso } \\
\text { hospitalario } \\
\text { dentro de las } \\
\text { posteriores ah } \\
\text { ictus }{ }^{b}\end{array}$ & $\begin{array}{l}\text { vida específica para la hemorragia } \\
\text { subaracnoidea aneurismática }\end{array}$ \\
\hline 39 & Baumann et al. & 2012 & $\begin{array}{l}\text { Luxemburgo } \\
\text { y Portugal }\end{array}$ & $\begin{array}{l}79 \text { Luxenbourg y } \\
48 \text { de Portugal }^{\mathrm{c}}\end{array}$ & $\begin{array}{l}\text { Analizar la satisfacción con la } \\
\text { vida dos años después del ictus y } \\
\text { su relación con la calidad de vida, } \\
\text { funcionalidad, variables } \\
\text { socieconómicas, satisfacción con } \\
\text { la vida y calidad de vida de los } \\
\text { cuidadores }\end{array}$ \\
\hline 40 & $\begin{array}{l}\text { Castellanos et } \\
\text { al. }\end{array}$ & 2012 & España & $\begin{array}{l}45 \\
\text { sobrevievientes a } \\
\text { un infarto } \\
\text { cerebral }^{\text {b }}\end{array}$ & $\begin{array}{l}\text { Estudiar la influencia de diversos } \\
\text { factores en la CVRS de los } \\
\text { pacientes tras un infarto cerebral, } \\
\text { con especial atención a los } \\
\text { trastornos psicopatológicos. }\end{array}$ \\
\hline 41 & $\begin{array}{l}\text { Chaiyawat \& } \\
\text { Kulkantrakorn }\end{array}$ & 2012 & Tailandia & $\begin{array}{l}60 \\
\text { sobrevivientes de } \\
\mathrm{ACV} \text { isquémico } \\
\text { reciente } \quad 30 \\
\text { grupo control y } \\
30 \text { de } \\
\text { rehabilitación) }{ }^{\mathrm{b}}\end{array}$ & $\begin{array}{llr}\text { Desarrollar } & \text { y } & \text { examinar la } \\
\text { efectividad de } & \text { un programa } \\
\text { individual de rehabilitación } & \text { de } \\
\text { domiciliaria de } 6 \text { meses } \\
\text { en pacientes con accidente } \\
\text { cerebrovascular isquémico sobre } \\
\text { la discapacidad y la calidad de } \\
\text { vida a los } 2 \text { años. }\end{array}$ \\
\hline 42 & Cramm et al. & 2012 & $\begin{array}{l}\text { Países bajos/ } \\
\text { Holanda }\end{array}$ & $\begin{array}{lr}251 \quad \text { diadas } \\
\text { (pacientes } \text { y } \\
\text { cuidadores) }^{\text {a }}\end{array}$ & $\begin{array}{l}\text { Identificar los factores asociados a } \\
\text { la } \mathrm{CV} \text { de pacientes con ictus y de } \\
\text { sus cuidadores }\end{array}$ \\
\hline 43 & $\begin{array}{l}\text { Gbiri y } \\
\text { Akinpelu }\end{array}$ & 2012 & Nigeria & $\begin{array}{l}65 \text { personas con } \\
\text { primer episodio } \\
\text { de accidente } \\
\text { cerebrovascula. }^{\text {b }}\end{array}$ & $\begin{array}{l}\text { Describir la CV de supervivientes } \\
\text { de accidente cerebrovascular } \\
\text { durante los primeros } 12 \text { meses } \\
\text { y determinar la influencia de } \\
\text { variables sociodemográficas. }\end{array}$ \\
\hline 44 & $\begin{array}{l}\text { López-Bastida } \\
\text { et al. }\end{array}$ & 2012 & $\begin{array}{l}\text { Islas } \\
\text { Canarias } \\
\text { España }\end{array}$ & $\begin{array}{l}448 \text { pacientes } \\
\text { con diagnóstico } \\
\text { de ictus. }{ }^{\text {c }}\end{array}$ & $\begin{array}{l}\text { Determinar el gasto económico y } \\
\text { CVRS en el primer, segundo y } \\
\text { tercer año después de sobrevivir a } \\
\text { ictus }\end{array}$ \\
\hline
\end{tabular}

Nota: ${ }^{\mathrm{a}}$ sobrevivientes en fase aguda; ${ }^{\mathrm{b}}$ sobrevivientes con $<6$ meses de recuperación, ${ }^{\mathrm{c}}$ sobrevivientes con $>$ de 6 meses de recuperación.

A partir de los objetivos, se identificaron 5 ejes temáticos abordados en los artículos de $\mathrm{CV}$ en sobrevivientes a ictus, siendo de mayor predominio el análisis de variables asociadas (77\%), seguido de la evaluación de la efectividad de programas de rehabilitación (9\%), la validación de instrumentos de medición (7\%), la descripción de la $\mathrm{CV}(5 \%)$ y finalmente los significados acerca de la experiencia de sobrevivir a un ictus (2\%) (Ver tabla 2).

En relación a las características de los participantes, el $100 \%$ de las investigaciones incluyó personas de 18 años en adelante, teniendo en cuenta que es la edad legalmente establecida en la mayoría de los países, para considerar a la persona con criterio para autorizar su participación en el estudio (p.ej Tang et al., 2013, 2014; Wong 
et al., 2013; Wu et al., 2014). Continuando con las características de las muestras, en el $55 \%$ de las pesquisas los participantes no se encontraban hospitalizados, mientras en el $34 \%$ de los estudios, en su mayoría transversales, los sobrevivientes estaban en alguna unidad hospitalaria; finalmente en el $14 \%$ de las investigaciones, las cuales eran longitudinales, se realizó la primera medición con personas hospitalizadas y el seguimiento se llevó a cabo cuando se encontraban en fase ambulatoria, lo cual puede generar un sesgo a la hora de realizar la comparación entre las mediciones. Adicionalmente, en la mayoría de los estudios (45\%), las valoraciones se realizaron en personas con más de 6 meses de evolución después del ictus, mientras en el 32\% de los artículos, participaron sobrevivientes con menos de 6 meses de evolución, seguido del $21 \%$ en el que los pacientes se encontraban en fase aguda y finalmente en el $5 \%$ se incluyeron sobrevivientes en diferentes fases de evolución.

\section{Instrumentos de evaluación}

También se realizó un análisis respecto a los instrumentos de evaluación utilizados por los investigadores, como se muestra en las tablas 3 y 4 .

Tabla 3

Instrumentos de evaluación de la CV de sobrevivientes a ictus

\begin{tabular}{lcc}
\multicolumn{1}{c}{ Instrumento/estrategia } & $\begin{array}{c}\text { Tipo de } \\
\text { instrumento }\end{array}$ & $\begin{array}{c}\text { Frecuencia } \\
\text { de uso }\end{array}$ \\
\hline The Short Form Health Survey (SF-36 y SF-12) & Genérica & $29 \%$ \\
Stroke Specific Quality of Life Scale (SS-QOL) & Específica & $25 \%$ \\
Euroqol (EQ-5D), & Genérica & $15 \%$ \\
Escala de Calidad de Vida para el ictus (ECVI - 38) & Específica & $6 \%$ \\
The World Health Organization Quality of Life (WHOQoL Bref) & Genérica & $6 \%$ \\
Newcastle Stroke-Specific Quality of Life Measure (NEWSQOL) & Específica & $2 \%$ \\
Assessment of Quality of Life (AQoL) & Genérica & $4 \%$ \\
Stroke Impact Scale (SIS) & Específica & $4 \%$ \\
Encuesta & - & $2 \%$ \\
Health-Related Quality of Life In Stroke Patients(HRQOLISP) & Específica & $2 \%$ \\
Entrevista semiestructurada & - & $2 \%$ \\
Subjective Quality of Life (SQOL) Scale & Genérica & $2 \%$ \\
\hline
\end{tabular}

De acuerdo con lo que se observa en la tabla 3, el 56\% de los investigadores utilizaron escalas genéricas para la evaluación de la $\mathrm{CV}$. El instrumento más utilizado fue la SF-36 a pesar del reporte de limitaciones como la presencia del efecto piso (porcentaje de muestra en el puntaje mínimo) y techo (porcentaje de muestra en la puntuación máxima) para algunas de las dimensiones, lo que indica que las respuestas de los evaluados estarían restringidas por el mismo test y no por las percepciones de los sujetos (Carod, 2004; Hobart et al.,2002; Krančiukaitė \& Rastenytė, 2006). Por su parte el SF12, a pesar de garantizar menor tiempo y costo en la aplicación, es menos preciso en la 
estimación de la salud individual y presenta dificultad para calcular los puntajes resumidos cuando queda un ítem sin respuesta (Pickard et al., 1999), estos mismos autores encontraron que el SF-12 reproduce las puntuaciones de resumen del SF-36 sin una pérdida sustancial de información en los pacientes con ictus capaces de dar autoinforme, sin embargo, las puntuaciones resumidas de salud mental de las evaluaciones proxy fueron altamente influenciadas por la edad. Cabe resaltar además que las escalas genéricas carecen de validez de contenido (Krančiukaitė \& Rastenytė, 2006), ya que no evalúan síntomas prevalentes de los sobrevivientes a ictus como hemiparesia, dependencia en actividades de la vida diaria, afasia, etc.

Respecto a las escalas específicas, la mayoría corresponde a auto reportes e incluyen ítems relacionados con los síntomas que se derivan del ictus. En la actualidad se están validando instrumentos específicos en diversos idiomas, así como en grupos con características particulares del ACV como el diagnóstico de aneurisma y hemorragia subaracnoidea (Boosman et al., 2010) y la validación y adaptación de instrumentos para pacientes con afasia (Raju \& Krishnan, 2015).

Se identificó también que en el estudio de Van Mierlo et al. (2015) se utilizó la encuesta como estrategia para medir el constructo, a partir de la pregunta ¿cuál es su calidad de vida en este momento?. Por su parte, Theeke et al. (2017) utilizaron la entrevista semiestructurada para indagar a profundidad la CV, sin embargo, los autores reconocen las limitaciones respecto a la generalización de los resultados.

Tabla 4

Instrumentos de evaluación de variables asociadas a CV de sobrevivientes a ictus

\begin{tabular}{lll}
\hline Variable & Instrumento & Frecuencia \\
Funcionalidad & Índice de Barthel & $39 \%$ \\
& Escala de Rankin Modificada & $23 \%$ \\
& Escala de Lawton y Brody & $9 \%$ \\
& Fugl-Meyer Assessment Of Sensorimotor Recovery After & $2 \%$ \\
& Stroke (FMA) & $27 \%$ \\
\hline Estatus & Escala de Evaluación Neurológica del Ictus (NIHSS) & $4 \%$ \\
Neurológico & Escala Neurológica Canadiense & $2 \%$ \\
& American Heart Association Stroke Outcome Classification & \\
\hline Variables & (AHA.SOC) & $32 \%$ \\
Psicosocial & Escala de Ansiedad y depresión Hospitalaria (HADS); Center & 3 \\
& for Epidemiological Studies Depression Scale (CES-D), \\
& Escala de Depresión de Hamilton, Geriatric Depression Scale & \\
& (GDS), Inventario de Depresión de Beck. Inventario & \\
\hline Rendimiento & Neuopsiquiátrico (NPI) & $20 \%$ \\
cognitivo & Mini Mental State Examination (MMSE) & $9 \%$ \\
\hline
\end{tabular}


La tabla 4 compendia los test y escalas frecuentemente utilizados para la evaluación de variables asociadas a CV de los sobrevivientes a ictus. Se identificó que los investigadores utilizan ampliamente instrumentos para valorar la funcionalidad del sujeto, debido a que es un síntoma altamente prevalente post ictus e imprescindible en el análisis de los resultados de tratamientos (Krančiukaite \& Rastenyte, 2006; Peña-Pitarch \& Tico-Falguera, 2012). De otra parte, se aplican también escalas que evalúan el estatus neurológico, con el propósito de determinar el nivel de conciencia y de comunicación, especialmente para realizar valoraciones que requieran autoreporte. Así mismo se encontró que los investigadores analizaron variables psicosociales como la depresión y la ansiedad, y no solo repercusiones motoras, como tradicionalmente ocurre (Espárrago, 2012).

Por otro lado, los investigadores adoptaron las puntuaciones obtenidas en los test de tamizaje cognitivo (MMSE y el MoCA) como criterio de inclusión en las muestras estudiadas, así mismo, se destaca que únicamente en dos estudios (Castellanos et al., 2012; Cumming et al., 2014) se utilizaron baterías más amplias y específicas para la evaluación de este dominio.

\section{Factores asociados a la CV post ictus}

Los estudios revisados evidencian la existencia de un grupo amplio y heterogéneo de variables que se asocian o predicen la calidad de vida de las personas sobrevivientes a ictus. Para un mejor análisis de las mismas se agruparon como se observa en la figura 2.

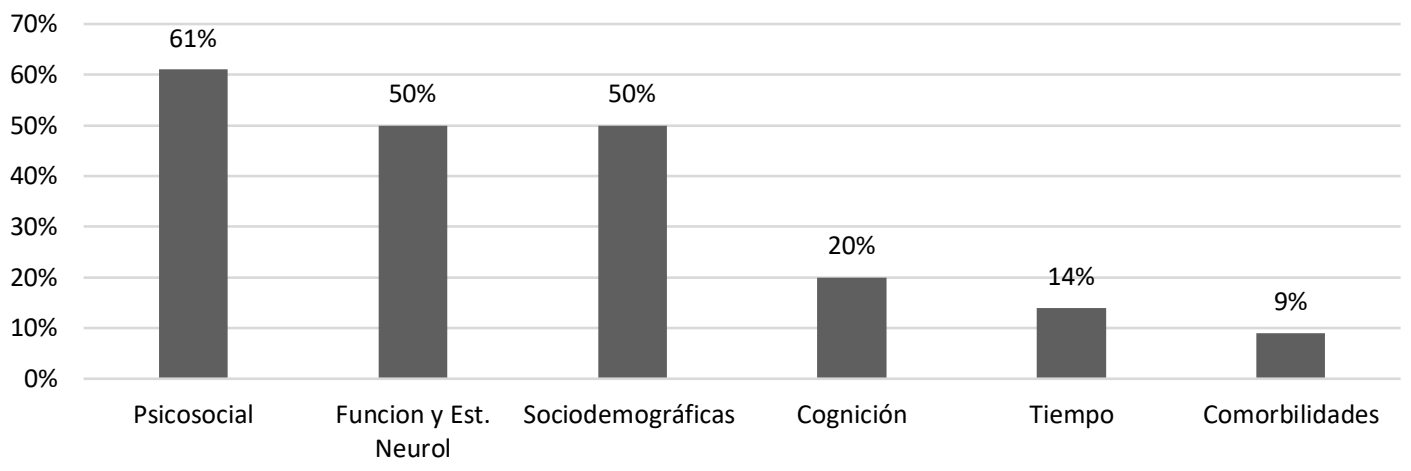

Figura 2. Distribución porcentual de las variables asociadas a la CV post ictus

Respecto a factores psicosociales, la depresión predominó significativamente (34\%) como predictor de CV post ictus, fue seguida de apoyo y soporte social o familiar (9\%), la ansiedad (4\%) y la participación psicosocial (4\%). Con menor frecuencia de 
estudio (2\%), las variables control percibido e incontinencia emocional, así como sentido de identidad post ictus y la dificultad de adaptación a la enfermedad. De otra parte, el $4 \%$ de los estudios refieren que puntuaciones altas en los ítems de depresión y ansiedad del Inventario Neuropsiquiátrico (NPI) son predictoras de la CV.

Del mismo modo, el 50\% de los estudios refieren que variables como el nivel de dependencia, la gravedad del ictus, las deficiencias neurológicas y la discapacidad al ingreso hospitalario predicen de manera significativa la $\mathrm{CV}$ de los sobrevivientes a ictus.

En cuanto a variables sociodemográficas, el $11 \%$ de los artículos revisados reportan que las mujeres perciben una mayor disminución de la CV en comparación a los hombres. También señalaron (20\% de los estudios) la relación inversamente proporcional entre la edad y CV post ictus, lo cual es coherente con lo propuesto por Pons et al. (2018). La asociación entre la CV y las variables bajo nivel educativo y el desempleo fue reportada en el $9 \%$ de los estudios. En cuanto al estado civil, se indica que ser casado o soltero puede asociarse a peor CV. De otra parte, el 9\% de los artículos revisados informaron que comorbilidades como la hipertensión arterial, artritis y enfermedades cardiacas, afectan la percepción de CV de los sobrevivientes a ictus.

En cuanto al rendimiento cognitivo, el buen desempeño en ésta área se describe como predictor de una mejor $\mathrm{CV}$ en las dimensiones física y de independencia. Particularmente, los procesos atencionales y la capacidad visoespacial, están fuertemente asociados con la calidad de vida post ictus (Cumming et al., 2014).

Por otro lado, variables como el tiempo de evolución (11\% de los estudios) y de estancia hospitalaria (2\%), fueron reportadas como predisponentes de CV. Otras variables menos estudiadas como la rehabilitación, la satisfacción con el cuidado, la recurrencia del evento, institucionalización, la localización de la lesión y el insomnio, también se reportaron como predictores de $\mathrm{CV}$ de pacientes con ictus.

\section{Limitaciones de los estudios descritas por los autores}

Finalmente, la limitación más referida por los estudios está relacionada con la muestra (59\%), tanto en la selección (heterogeneidad, exclusión de participantes con secuelas más graves o muestreos no probabilísticos) como en el tamaño (no representativo); a pesar de lo expuesto por los autores, dichas limitaciones son difícilmente corregibles, debido al acceso y a las características de la población. Por otro lado, refieren como limitante, la falta de indagación de otros factores que podrían estar 
asociados a la CV como lo son las variables psicofisiológicas y/o psicosociales, y el tamaño, gravedad, área y tiempo transcurrido de la lesión. Adicionalmente el 18\% de las investigaciones indican que el diseño transversal restringe el análisis explicativo de las relaciones multivariables.

\section{Discusión}

La CV está enmarcada en la percepción de bienestar que realiza cada persona, tanto de elementos objetivos como subjetivos, de las distintas dimensiones de su vida (Urzúa \& Caqueo, 2012), a pesar del alto grado de subjetividad que comprende el constructo, fue reducido el número de estudios cualitativos y mixtos hallados en la revisión, evidenciándose la necesidad de investigaciones que permitan ampliar la comprensión del fenómeno a partir de metodologías cuantitativas y cualitativas.

Por otra parte, se identifica la necesidad de proponer y evaluar la efectividad de programas de rehabilitación en sobrevivientes a ictus, en tanto la mayoría de artículos son de carácter observacional, centrados en la identificación de factores asociados a la calidad de vida, y muy pocos permiten identificar la validez de las intervenciones; adicionalmente los procesos de rehabilitación reportados en los artículos, se fundamentan en la recuperación de las habilidades motoras, las cuales tuvieron una repercusión positiva en la CV de los participantes, no obstante, surge la necesidad de ampliar los estudios para identificar la eficacia de otros procesos interventivos, tales como la rehabilitación cognitiva y el acompañamiento psicoterapéutico (Cicerone et al., 2005), de tal manera que se generen procesos integrativos basados en la evidencia.

Es importante considerar que más del $50 \%$ de los estudios rastreados fueron de corte transversal, por lo cual, autores como De Souza et al., (2013) plantean que dicho diseño limita el estudio, ya que, la relación entre las variables puede cambiar con el tiempo o podrían haber sido influenciada por factores distintos a los que se consideraron en la investigación, y por tanto, los diseños longitudinales serían útiles para documentar los cambios en el comportamiento de estas variables y en las asociaciones entre ellas. En esta misma línea, Jönsson et al. (2014), refieren que el tiempo de seguimiento en los estudios longitudinales es limitado y señalan que hay pocas investigaciones con un rastreo mayor a 5 años después del ictus.

Uno de los resultados más relevantes de este estudio es que la afectación de la funcionalidad y el estado neurológico consecuente, son variables altamente relacionadas con la CV. La depresión es otra variable que se estudia ampliamente como posible 
predictor de $\mathrm{CV}$, ya que es uno de los principales factores que limitan la recuperación de los pacientes (Espárrago et al., 2012), ya sea como producto de una reacción psicológica por el estado funcional actual (Aguilar et al., 2009), o como secuela de orden neurobiológico por lesiones fronto-subcorticales del lado izquierdo del cerebro (Espárrago et al., 2012).

Los estudios revisados también señalan la relevancia de otras variables psicosociales en la CV del paciente con ictus como el apoyo social y familiar, la interacción social y la presencia de síntomas neuropsiquiátricos, sin embargo, el reporte de estudios frente a estas variables es menor, en comparación con los que analizan la depresión. Cabe resaltar que aún no se conocen los mecanismos claros bajo los que aparecen síntomas como la ansiedad y que los estudios aún se encuentran en sus primeras etapas (Schöttke \& Giabbiconi 2015), por lo cual se espera que futuras investigaciones analicen los factores antes mencionados para lograr un abordaje integral de la CV de los sobrevivientes a ictus.

Por otro lado, predominaron las pesquisas realizadas con sobrevivientes que se encontraban en fase crónica; a diferencia de lo hallado por Durá et al., (2011), quienes plantean que la mayor atención de los estudios se centra en la fase aguda, en comparación con la cantidad de investigaciones realizadas en fase subaguda o crónica. En esta misma línea, los estudios analizados evidencian la influencia de la variable tiempo de evolución del ictus en la predicción de la CV, este dato es coherente con la conceptualización del constructo, ya que se trata de un fenómeno dinámico, en donde la percepción puede modificarse con el tiempo, debido a las experiencias vividas y a cambios que se pueden presentar en el contexto, razón por la cual se requieren estudios longitudinales para validar los resultados.

En cuanto a lo cognitivo se encontraron estudios que utilizaron las puntuaciones de instrumentos de screening (MMSE y MoCA) como criterio de exclusión, lo que supone un problema de investigación debido a que no se valoraron a las personas con puntuaciones bajas en dichos test, sin embargo, esto no significa la inexistencia de la afectación en $\mathrm{CV}$ en personas con bajo rendimiento cognitivo, por lo que se requiere de estrategias de evaluación adecuadas para este grupo poblacional.

Así mismo, pocos estudios incluyeron baterías amplias para la evaluación de los déficits cognitivos y su consecuente efecto sobre la $\mathrm{CV}$, a pesar de existir evidencias de la influencia de la inatención, el síndrome disejecutivo y disminución de la velocidad de procesamiento en la resolución de tareas de la vida diaria (Gramstad et al., 2011; Mitchell 
et al., 2010), lo que sugiere utilizar instrumentos de evaluación que permitan indagar de manera detallada el procesamiento cognitivo de los sobrevivientes a ictus, además de la aplicación de test de tamizaje.

En cuanto a la evaluación de la CV post ictus, el SF-36 es el instrumento más utilizado, sin embargo, existen otros test específicos que incluyen la valoración de dimensiones acordes con las secuelas más frecuentes post ictus (ver tabla 3), entre las que se incluyen la afectación en la dimensión cognitiva, la movilidad y el desarrollo de actividades de la vida diaria. En este sentido se debe seleccionar la estrategia de evaluación más pertinente, a partir del análisis de las condiciones de los participantes.

Teniendo en cuenta el número de bases de datos revisadas en el presente manuscrito, se muestra un panorama parcial de las investigaciones sobre el tema de interés, develando algunos aspectos relevantes que serían necesarios considerar a la hora de estudiar la $\mathrm{CV}$ en sobrevivientes a ictus, tales como la inclusión de diseños observacionales longitudinales con mayor tiempo de seguimiento, la evaluación de procesos de rehabilitación, la inclusión de participantes con edades inferiores a los 18 años, la medición de la CV con escalas específicas, así como profundizar en variables cognitivas, psicológicas y del proceso de cuidado. 


\section{Referencias}

Aguilar, H., Olivera, J., Benabarre, S., \& Pelegrín, C. (2009). Psicopatología del accidente cerebrovascular: el estado de la cuestión. Psicogeriatría, 1, 23-35. https://www.viguera.com/sepg/pdf/revista/0101/0101_23_35.pdf

Andrew, N. E., Kilkenny, M. F., Lannin, N. A., \& Cadilhac, D. A. (2016). Is healthrelated quality of life between 90 and 180 days following stroke associated with long-term unmet needs? Quality of Life Research, 25(8), 2053-2062. http://dx.doi.org/10.1007/s11136-016-1234-5

Baumann, M., Lurbe, K., Leandro, M., \& Chau, N. (2012). Life satisfaction of twoyear post-stroke survivors: Effects of socio-economic factors, motor impairment, newcastle stroke-specific quality of life measure and world health organization quality of life - bref of informal caregivers in luxembourg and a rural area in portugal. Cerebrovascular Diseases, 33(3), 219-30. http://dx.doi.org//10.1159/000333408

Bernal, C. (2010). Metodología de la investigación. administración, economía, humanidades y ciencias sociales. Editorial Pearson

Boosman, H., Passier,P., Visser-Meily, J.M., \& Rinquel, G. (2010). Validation of the Stroke Specific Quality of Life scale in patients with aneurysmal subarachnoid haemorrhage. Journal of Neurology, Neurosurgery \& Psychiatry, 81, 485-489. http://dx.doi.org/10.1136/jnnp.2009.184960

Bradford-Hill, A. (1992). Ambiente y enfermedad: ¿asociación o causación? Boletín de la Oficina Sanitaria Panamericana, 113, 233-242. http://www.redalyc.org/articulo.oa?id=21419850015

Carod, F. (2004). Escalas específicas para la evaluación de la calidad de vida en el ictus. Revista de Neurología, 39 (11), 1052-1062. http://www.sld.cu/galerias/pdf/sitios/rehabilitacion/calidad ictus.pdf

Castellanos, F., Hernández, J., Zurdo, M., Rodríguez, B., García C., et al., (2012). Trastornos psicopatológicos y calidad de vida en el infarto cerebral. Neurología, 27(2), 76-82, http://dx.doi.org/10.1016/j.nrl.2011.04.004

Chaiyawat, P., \& Kulkantrakorn, K. (2012). Effectiveness of home rehabilitation program for ischemic stroke upon disability and quality of life: A randomized controlled trial. Clinical Neurology and Neurosurgery, 114(7), 866-70. http://dx.doi.org/10.1016/j.clineuro.2012.01.018

Chou, C. (2015). Determinants of the health-related quality of life for stroke survivors, Journal of Stroke and Cerebrovascular Diseases, 24(3), 655-662. http://dx.doi.org/10.1016/j.jstrokecerebrovasdis.2014.10.022

Chuluunbaatar, E., Chou, Y.J., \& Pu, C. (2016). Quality of life of stroke survivors and their informal caregivers: A prospective study. Disability Health Journal, 9(2), 306-12. http://dx.doi.org/10.1016/j.dhjo.2015.10.007

Cicerone, K.D., Dahlberg, C., Malec, J.F., Langenbahn, D.M., Felicetti, T., Kneipp, S., Ellmo, W., Kalmar, K., Giacino, J., Harley, J.P., Laatsch, L., Morse, P.A., \& Catanese J. (2005). Evidence-based cognitive rehabilitation: updated review of the literature from 1998 through 2002. Archives of Physical Medicine and Rehabilitation, 86, 1681-1692. http://dx.doi.org/10.1016/j.apmr.2005.03.024

Cortez, M., Wilder, M., McFadden, M., \& Majersik, J. (2014). Quality of life after intra-arterial therapy for acute ischemic stroke. Journal of Stroke and Cerebrovascular Diseases, 23(7), 1890-1896 http://dx.doi.org/10.1016/j.jstrokecerebrovasdis.2014.02.012 
Cramm, J. M., Strating, M. M., H., \& Nieboer, A. P. (2012). Satisfaction with care as a quality-of-life predictor for stroke patients and their caregivers. Quality of Life Research, 21(10), 1719-25. http://dx.doi.org/10.1007/s11136-011-0107-1

Crichton, S. L., Bray, B. D., McKevitt, C., Rudd, A. G., \& Wolfe, C. D. A. (2016). Patient outcomes up to 15 years after stroke: Survival, disability, quality of life, cognition and mental health. Journal of Neurology, Neurosurgery and Psychiatry, 87(10), 1091-1098. http://dx.doi.org/10.1136/jnnp-2016-313361

Cruz-Cruz, C., Martinez-Nuñez, J., Perez, M., Kravzov-Jinich, J., Ríos-Castañeda, C., \& Altagracia-Martinez, M. (2013). Evaluation of the Stroke-Specific Quality-Of-Life (SSQOL) scale in México: a preliminary approach. Value in Health Regional Issues, 2(3), 392-397. http://dx.doi.org/10.1016/j.vhri.2013.04.002

Cumming, T., Brodtmann, A., Darby, D., \& Bernhardt, J. (2014). The importance of cognition to quality of life after stroke. Journal of Psychosomatic Research, 77(5), 374-379. http://dx.doi.org/10.1016/j.jpsychores.2014.08.009

Dąbrowska-Bender, M., Milewska, M., Gołąbek, A., Duda-Zalewska, A., \& Staniszewska, A. (2017). The impact of ischemic cerebral stroke on the quality of life of patients based on clinical, social and psychoemotional factors. Journal of Stroke and Cerebrovascular diseases, 26(1), 101-107 http://dx.doi.org/10.1016/j.jstrokecerebrovasdis.2016.08.036

De Lima, M., Ferreira, J., Okino, N \& Pereira, L. (2014). Quality of life of individuals with stroke and their caregivers in a city of Triângulo Mineiro. Revista brasileña de epidemiología., 17(2), 453-64 http://dx.doi.org/10.1590/1809-4503201400020013ENG

De Souza, A., da Costa Rocha, M., Teixeira, A., Dias, J., Pereira de Sousa, L., \& Pereira, M. (2012). Depressive symptoms and disability in chagasic stroke patients: Impact on functionality and quality of life. Journal of the Neurological Sciences, 324 (1-2), 34-37 http://dx.doi.org/10.1016/j.jns.2012.09.022

Guajardo, V., Terroni, L., de Freitas, M., dos Santos, M., Tinone, G., Scaff, M., Losifescu, D., Souza, M., \& Fraguas, R. (2015). The influence of depressive symptoms on quality of life after stroke: a prospective study. Journal of Stroke and Cerebrovascular Diseases, 24(1), 201-209. http://dx.doi.org/10.1016/j.jstrokecerebrovasdis.2014.08.020

Dhamoon, M. S., McClure, L. A., White, C. L., Lakshminarayan, K., Benavente, O. R., \& Elkind, M. S. V. (2015). Long-term disability after lacunar stroke: Secondary prevention of small subcortical strokes. Neurology, 84(10), 10021008. http://dx.doi.org/10.1212/WNL.0000000000001331

Durá, M., Molleda, M., García, C., Mallol, J., Calderon, V. (2011). Factores pronósticos en el ictus. De la fase aguda a los tres años. Rehabilitacion, 45(1) 18-23. http://dx.doi.org/10.1016/j.rh.2010.09.004

Ellis, C., Grubaugh, A., \& Egede, L. (2013). Factors associated with sf-12 physical and mental health quality of life scores in adults with stroke. Journal of Stroke and Cerebrovascular Diseases, 22(4), 309-317. http://dx.doi.org/10.1016/j.jstrokecerebrovasdis.2011.09.007

Elsevier. (2020). Qué es ScienceDirect. https://es.qwe.wiki/wiki/ProQuest

Espárrago, G., Castilla-Guerra, L., Fernández, M., Ruiz, S., \& Jiménez, M. (2015). Depresión post ictus: una actualización. Neurologia, 30, 23-31, http://dx.doi.org/10.1016/j.nrl.2012.06.008 
Ezeugwu, V., \& Manns, P. (2017). Sleep duration, sedentary behavior, physical activity, and quality of life after inpatient stroke rehabilitation. Journal of Stroke and Cerebrovascular Diseases, 26(9), 2004-2012, http://dx.doi.org/ 10.1016/j.jstrokecerebrovasdis.2017.06.009

GBD 2016 Stroke Collaborators. (2019). Global, regional, and national burden of stroke, 1990-2016: a systematic analysis for the Global Burden of Disease Study 2016. The Lancet. Neurology, 18(5), 439-458. https://doi.org/10.1016/S1474-4422(19)30034-1

Gillard, P. J., Sucharew, H., Kleindorfer, D., Belagaje, S., Varon, S., Alwell, K., Moomaw, C., Woo, D., Khatri, P., Flaherty, M., Adeoye, O., Ferioli, S., \& Kissela, B. (2015). The negative impact of spasticity on the health-related quality of life of stroke survivors: A longitudinal cohort study. Health and Quality of Life Outcomes, 30 (1), 18-24 http://dx.doi.org/10.1186/s12955015-0340-3

González-Gómez, F., Pérez-Torre, P., DeFelipe, A., Vera, R., Matute, C., CruzCulebras, A., Álvarez-Velasco, R., \& Masjuan, J. (2016). Stroke in young adults: Incidence rate, risk factors, treatment and prognosis. Revista Clínica Española., 216(7), 345-351. http://dx.doi.org/10.1016/j.rce.2016.05.008

Gramstad, A., Aarsland, D., \& Naess, H. (2011). Cognitive profile of elderly patients with mild stroke. Dementia and Geriatric Cognitive Disorders Extra., 1(1), 409-417 http://dx.doi.org/10.1159/000331675

Haghgoo, H., Pazuki, E., \& Hosseini, A. \& Rassafiani, M. (2013). Activities of daily living and quality of life in patients with stroke. Journal of the Neurological Sciences, 328, (1-2), 87-91. http://dx.doi.org/10.1016/j.jns.2013.02.027

Hobart, J., Williams, L., Moran, K., \& Thompson, A. (2002). Quality of life measurement after stroke uses and abuses of the SF-36. Stroke, 33, 13481356. http://dx.doi.org/10.1161/01.STR.0000015030.59594.B3

Hohmann, C., Radziwill, R., Klotz, J. M., \& Jacobs, A. H. (2010). Health-related quality of life after ischemic stroke: The impact of pharmaceutical interventions on drug therapy (pharmaceutical care concept). Health and Quality of Life Outcomes, 8(59), 1-7. http://dx.doi.org/10.1186/1477-7525-8$\underline{59}$

Huang, Y.H., Wu, C.Y., Lin, K.C., Hsieh, Y.W., Snow, W.M., \& Wang, T.N. (2013). Determinants of change in stroke-specific quality of life after distributed constraint-induced therapy. American Journal of Occupational Therapy, 67(1), 54-63. http://dx.doi.org/10.5014/ajot.2013.004820

Jaracz, K., Grabowska-Fudala, B., Górna, K., \& Kozubski, W. (2014). Consequences of stroke in the light of objective and subjective indices: a review of recent literature. Neurologia i Neurochirurgia Polska, 48(4):280-286. http://dx.doi.org/10.1016/j.pjnns.2014.07.004

Jönsson, A.N., Delavaran, H., Iwarsson, S., Ståhl, A., Norrving, B., \& Lindgren, A. (2014). Functional status and patient-reported outcome 10 years after stroke: the lund stroke register. Stroke, 45(6), 1784-1790. http://dx.doi.org/10.1161/STROKEAHA.114.005164

Karube, N., Sasaki, A., Hondoh, F., Odagiri, C., Hagii, J., Seino, S., Yasujima, M., \& Osanai, T. (2016). Quality of life in physical and psychological health and social environment at posthospitalization period in patients with stroke. Journal of Stroke and Cerebrovascular Diseases, 25(10), 2482-2487. http://dx.doi.org/10.1016/j.jstrokecerebrovasdis.2016.06.022 
Khalid, W., Rozi, S., Tazeen, S. A., Azam, I., Mullen, M. T., Illyas, S., Un-Nisa, Q., Soomro, N., \& Kamal, A. K. (2016). Quality of life after stroke in pakistan. BMC Neurology, 16, 250. http://dx.doi.org/10.1186/s12883-016-0774-1

Kim, S. Y., Kim, J. M., Stewart, R., Kang, H. J., Kim, S. W., Shin, I. S., Park, M. S., Cho, K. H., \& Yoon, J. S. (2013). Influences of personality traits on quality of life after stroke. European Neurology, 69(3), 185-92. http://dx.doi.org/10.1159/000345699

Kim, W. H., Jung, H. Y., Choi, H. Y., Park, C. H., Kim, E. S., Lee, S. J., Ko, S. H., Kim, S. Y., \& Joa, K. L. (2017). The associations between insomnia and health-related quality of life in rehabilitation units at 1 month after stroke. Journal of Psychosomatic Research, 96, 10-14 http://dx.doi.org/10.1016/j.jpsychores.2017.02.008

Krančiukaite, D \& Rastenyte, D. (2006). Measurement of quality of life in stroke patients. Medicina (Kaunas); 42(9), 709-716. http://medicina.1smuni.lt/med/0609/0609-03e.htm

Leno, C., Holguín, M., Hidalgo, N., Rodriguez-Ramos, M., \& Lavado, J. (2016). Calidad de vida relacionada con la salud en personas sobrevivientes a un ictus a largo plazo. Revista Científica de la Sociedad Española de Enfermería Neurológica, 44, 9-15 https://doi.org/10.1016/j.sedene.2015.12.002

López-Bastida, J., Oliva Moreno, J., Worbes Cerezo, M., Perestelo Perez, L., Serrano-Aguilar, P., \& Montón-Álvarez, F. (2012). Social and economic costs and health-related quality of life in stroke survivors in the Canary Islands, Spain. BMC Health Services Research, 12, 315. https://doi.org/10.1186/1472-6963-12-315

McDonnell, M. N., Mackintosh, S. F., Hillier, S. L., \& Bryan, J. (2014). Regular group exercise is associated with improved mood but not quality of life following stroke. Peer J, 27(2), 1-10. http://dx.doi.org/10.7717/peerj.331

Mesa, Y., Hernández, T., \& Parada, Y. (2017). Factores determinantes de la calidad de vida en pacientes sobrevivientes a un ictus. Revista Habanera de Ciencias Médicas, 16(5), 735-750. http://scielo.sld.cu/pdf/rhcm/v16n5/rhcm07517.pdf

Mitchell, A., Kemp, S., Benito-León, J., \& Reuber, M. (2010). The influence of cognitive impairment on health-related quality of life in neurological disease. Acta Neuropsychiatrica, 22, 2-13. https://doi.org/10.1111/j.16015215.2009.00439.x

Peña-Pitarch, E., \& Tico-Falguera, N. (2012). Assessment of damage in affected persons with neurological sequelae. Trauma Fund MAPFRE, 23 (4), 247-252. http://www.mapfre.com/fundacion/html/revistas/trauma/v23n4/docs/Articulo 8.pdf

Persson, J., Levin, L., Holmegaard, L., Redfors, P., Jood, K., Jern, C., Blomstrand, C., \& Forsberg-Warleby, G. (2017). Stroke survivors long-term QALYweights in relation to their spouses QALY-weights and informal support: A cross-sectional study. Health and Quality of Life Outcomes, 15, 150. https://doi.org/10.1186/s12955-017-0724-7

Pickard, S., Johnson, J.A., Penn, A., Lau, F., \& Noseworthy, T. (1999). Replicability of SF-36 summary scores by the SF-12 in stroke patients. Stroke 30(6), 12131217. https://doi.org/10.1161/01.STR.30.6.1213

Pinedo, S., Sanmartín, V., Zaldibar, B., Erazo, P., Miranda, M., Tejada, P., Lizarraga, N., Aycart, J., Gamio, A., \& Bilbao, A. (2016). Calidad de vida a los 6 meses tras un ictus. Rehabilitación, 50(1), 5-12, https://doi.org/10.1016/j.rh.2015.08.002 
Pons, S., Delgado, V., Oliva, M., Tamayo, Y., \& González, J. (2018). Apreciación de la calidad de vida en el adulto mayor. Revista Médica Granma, 22(1), 39-50. http://www.revmultimed.sld.cu/index.php/mtm/article/view/777/1105

Proquest. (2020). Proquest libguides. https://proquest.libguides.com/hamcollection

Rachpukdee, S., Howteerakul, N., Suwannapong, N., Tang-Aroonsin, S. (2013). Quality of Life of Stroke Survivors: A 3-Month Follow-up Study. Journal of Stroke and Cerebrovascular Diseases, the official journal of National Stroke Association, 22(7), e70-e78 https://doi.org/10.1016/j.jstrokecerebrovasdis.2012.05.005

Raju, R., \& Krishnan, G. (2015). Adaptation and validation of stroke-aphasia quality of life (SAQOL-39) scale to Malayalam. Annals of Indian Academy of Neurology, 18(4), 441-444. http://doi.org/10.4103/0972-2327.160068

Robles, A., Rubio, B., De La Rosa, E., Vanessa, E., \& Nava, A. (2016).

Generalidades y conceptos de calidad de vida en relación con los cuidados de salud. El Residente, 11(3), 120-125. http://www.medigraphic.com/pdfs/residente/rr-2016/rr163d.pdf

Sarfo, F.S., Jenkins, C., Singh, A., Owolabi, M., Ojagbemi, A., Adusei, N., Saulson, R., \& Ovbiagele, B. (2017). Post-stroke depression in Ghana: Characteristics and correlates. Journal of the Neurological Sciences, 379, 261-265. https://doi.org/10.1016/j.jns.2017.06.032

Schöttke, H., \& Giabbiconi, C. M. (2015). Post-stroke depression and post-stroke anxiety: prevalence and predictors. International Psychogeriatrics, 27(11):1805-1812. https://doi.org/10.1017/S1041610215000988

Soriano, A.P., Coarasa, A., Reigada, P., Solano, V. (2013). Empleo de la escala de calidad de vida para el ictus (ECVI-38) para cuantificar y medir las consecuencias de un ictus. Relación con variables demográficas y clínicas. Rehabilitación (Madr), 47(4), 213-222, https://doi.org/10.1016/j.rh.2013.06.001.

Tang, W.K., Lau, C.G., Mok, V., Ungvari, G.S., \& Wong, K.S. (2013). , Impact of Anxiety on Health-Related Quality of Life After Stroke: A Cross-Sectional Study. Archives of Physical Medicine and Rehabilitation, 47(4), 213-222. https://doi.org/10.1016/j.rh.2013.06.001

Tang, W.K., Lau, C.G., Mok, V., Ungvari, G.S., \& Wong, K.S. (2014). Apathy and health-related quality of life in stroke. Archives of Physical Medicine and Rehabilitation, 95(5), 857-861, https://doi.org/10.1016/j.apmr.2013.10.012.

The World Health Organization Quality of Life Assessment (WHOQOL): development and general psychometric properties. (1998). Social Science \& Medicine (1982), 46(12), 1569-1585. https://doi.org/10.1016/s02779536(98)00009-4

Theeke, L.A., Lucke-Wold, A. N., Mallow, J.A., \& Horstman, P. (2017). Life after stroke in Appalachia. International Journal of Nursing Sciences, 4, 105-111 https://doi.org/10.1016/j.ijnss.2017.02.005

Tran, P. L., Leigh Blizzard, C., Srikanth, V., Hanh, V. T., Lien, N.T., Thang, N.H., \& Gall, S. L. (2015). Health-related quality of life after stroke: Reliability and validity of the duke health profile for use in Vietnam. Quality of Life Research, 24(11), 2807-2814.

https://doi.org/10.1007/s11136-015-1016-5

Urzúa, A. (2010). Calidad de vida relacionada con la salud: Elementos conceptuales. Revista Médica de Chile, 138(3), 358-365. https://doi.org/10.4067/S0034$\underline{98872010000300017}$ 
Urzúa, MA., \& Caqueo-Urízar, A. (2012). Calidad de vida: una revisión teórica del concepto. Terapia psicológica., 30(1), 61-71. https://doi.org/10.4067/S0718$\underline{48082012000100006}$

Van Mierlo, M.L., van Heugten, C.M., Post, M., de Kort, P., \& Visser-Meily, J. (2015). Life satisfaction post stroke: The role of illness cognitions. Journal of Psychosomatic Research, 79, 137-142. https://doi.org/10.1016/j.jpsychores.2015.05.007

Wu, X., Min, L., Cong, L., Jia, Y., Liu, C., Zhao, H., Liu, P., \& Luo, Y. (2014). Sex differences in health-related quality of life among adult stroke patients in Northeastern China. Journal of Clinical Neuroscience, 21(6), 957-961. https://doi.org/10.1016/j.jocn.2013.08.030 